\title{
Comparison between Simulations and Theory: where we stand
}

\author{
Joe J. Monaghan ${ }^{1}$ \\ ${ }^{1}$ School of Mathematical Sciences \\ Vic 3800, Monash University, Australia \\ email: joe.monaghan@monash.edu
}

\begin{abstract}
In this paper I relate progress in the simulation of star formation to a remarkable early (1962) paper by Fowler and Hoyle who analyzed star formation in terms of the relevant energies starting from the formation of a large $(100 \mathrm{pc})$ cloud and ending with the final mass distribution of the stars. The way in which modern simulations have clarified and corrected the Fowler-Hoyle picture, and areas where we could improve our simulations, are discussed.
\end{abstract}

Keywords. Star formation

\section{Introduction}

The aim of a theory of star formation is to predict the mass, internal rotation, composition, and magnetic fields of the observed stars. I take the essentials of a theory to be the equations that describe the system and their subsequent solution. There is general agreement that the relevant equations are those of gas dynamics with gravity and magnetic fields (at the very least in the MHD approximation), the chemistry of transformations between atoms and molecules, radiative transfer and the effects of grain formation and destruction. Most of the gas in the galaxy is turbulent and we could reasonably expect that ultimately we will need a turbulence theory to provide sub-grid effects.

We can gauge the progress made towards achieving our aims by comparing it with the remarkable paper of Willy Fowler and Fred Hoyle (1962). In that paper Fowler and Hoyle recognized the need for a theory to show:

- That a large $(100 \mathrm{pc})$ cloud of gas in our galaxy could collapse despite the shear, magnetic fields, cosmic rays, and turbulence.

- That such a cloud would continue to collapse, and the Jeans mass continue to decrease, allowing fragmentation.

- That the fragmentation would cease when the opacity was sufficiently large for the compressional heat to be retained and, at that stage, the fragments would have masses comparable to the observed masses.

Because the computers available to Fowler and Hoyle were incapable of simulating the gas dynamics relevant to star formation, Fowler and Hoyle used approximate energy arguments to estimate the importance of the various physical effects. Furthermore, they were not aware, or were unable, to include the effects of accretion, and they were not able to predict the formation of binary systems. Nevertheless, much of what they deduced is relevant to the aims of present conference.

The initial step of the Fowler-Hoyle theory was the formation of clouds from the galactic material. They estimated the density required to overcome the rotation of the galaxy, and with this density, and estimates of the magnetic fields and turbulence, they estimated the energy contributions from gravitational, magnetic fields, turbulence and cosmic rays. This enabled them to assert that clouds could collapse and that once they did, further 
fragmentation was inevitable, though the explanation of the way this occurred was hazy. Contributions to this conference have described simulations of large cloud formation in a galaxy in the absence of magnetic fields along the lines envisaged by Fowler and Hoyle. These simulations have the potential to provide realistic initial conditions for the subsequent fragmentation of the clouds.

In the last several years extensive simulations, some of which have been described at this conference, have followed the evolution of large clouds that fragment to produce stellar systems. The most recent of these, which use both SPH and AMR methods, also include radiative transfer and magnetic fields. This work has clarified the conjectures of Fowler and Hoyle concerning the fragmentation of clouds forming in the galaxy.

In order to follow the dynamics of a fragmenting cloud for as long as possible sink particles are used to replace very high condensations of gas. As a consequence, the full range of phenomena from cloud to stars, have not yet been simulated though it has been possible to show that mass functions similar to those observed are predicted from the simulations. A question that was not taken up by Fowler and Hoyle is the effect of feed-back from newly formed stars sufficiently massive to become supernova. They inject energy and generate turbulence, and they change the metal composition which in turn affects radiative transfer. Studies of this feed-back are in their infancy.

In summary the current simulations allow us first to follow the early stages of clouds forming in the galaxy, second to use a variety of initial conditions to simulate fragmentation, and third to predict the mass function of the stars that are produced by the fragmentation. They do not allow us to start with a galaxy and follow the evolution to stars, and they do include the detailed effects of supernova and other possible feed back mechanisms. There are however, a number of questions that we might ask concerning the accuracy and relevance of simulations, and I will discuss some of these below.

\section{Questions concerning the accuracy and relevance of simulations}

The following are questions we need to answer in order to establish that the results we get from the simulations of star formation can be believed.

- Do we have the right equations?

- Are we solving them correctly?

- Do we have the right initial conditions?

- Do we have the right boundary conditions?

- Is feed-back from rapidly evolving stars included correctly?

- Are we treating diffusion, mixing and turbulence correctly?

- Is dust being included correctly?

Some of these questions are easy to answer, and they vary in importance. We believe we know the right equations to solve though this is done with varying degrees of approximation. The algorithms currently used fall into two large classes. The first being finite difference methods, conveniently but inaccurately labelled as AMR, the second are particle methods collectively described as SPH. In practice AMR is a handy name to tell us that the mesh of a finite difference scheme is subdivided to achieve better resolution, but the actual finite difference scheme can vary. Some of them are very good, and others rather poor. In this conference Krumholz, Tyssier, Norman and others show impressive results obtained with AMR methods. SPH is a general particle method and the actual algorithm depends on the kernels used, the rules for the spatial gradients, and the time stepping algorithm. Some are better than others. The results of Bate, Price, Bonnell, Springel, Whitworth, Klessen and others at this conference have shown a range of impressive results from carefully tested $\mathrm{SPH}$ algorithms. 
As an example of the different treatments of the SPH equations we can consider the $\mathrm{SPH}$ pressure force term which can be written in the form (Monaghan(2005))

$$
\frac{\nabla P}{\rho} \rightarrow \sum_{b} m_{b}\left(\frac{P_{a}}{\Omega_{a} \rho_{a}^{2}}+\frac{P_{b}}{\Omega \rho_{b}^{2}}+\Pi_{a b}\right) \nabla_{a} W_{a b},
$$

where $m_{a}, P_{a}$, and $\rho_{a}$, are the mass, pressure, density of particle $a, \Pi_{a b}$ provides viscosity, and $W_{a b}$ is the SPH kernel. The function $\Omega$ takes account of the variation of the resolution with density. If this is applied to an ideal gas at constant pressure in a periodic box, and the SPH particles are placed on a grid of squares, it will be found that the particles will move around their equilibrium positions with an energy that is about 0.01 the thermal energy. This occurs because the SPH algorithm has some of the attributes of molecular dynamics and the particles seek out the lowest energy configuration. In two dimensions this low energy state is close to a grid of squares but the preferred positions approximate a grid of hexagons. In practice this initial motion is entirely negligible, and for a star formation problem it is only 0.01 of the thermal energy. However, some authors find it tempting to write the SPH equations so that a constant pressure configuration will remain at rest. One way to do this is to write the previous equation as

$$
\frac{\nabla P}{\rho} \rightarrow \sum_{b} m_{b}\left(\frac{\left(P_{b}-P_{a}\right)}{\rho_{b}^{2}}+\Pi_{a b}\right) \nabla_{a} W_{a b},
$$

where we have dropped the $\Omega$ since they do not affect our argument. There are at least two problems with this equation. First it means that gas at constant pressure in a tube with the ends open, and connected to a vacuum, will not move even though it should. If, on the other hand, (2.1) is used the SPH particles near the vacuum will feel a pressure force from their neighbours in the interior of the tube and they will move out of the tube. The same thing happens with a finite difference calculation. The second problem is that the pressure force in (2.2) doesn't conserve momentum. As a consequence two particles initially at rest, but with different pressures will be accelerated in the same direction with increasing speed. The final speed is only limited by collisions or the transfer of heat between the particles which cause the pressures to become equal whereupon the rapidly moving particles will slow down and stop. If (2.1) is used the two SPH particles will move apart with equal and opposite velocities reproducing, though with very poor resolution, the expansion of a parcel of gas in a vacuum.

Because some researchers believe SPH cannot simulate problems where two fluids with very different densities are in contact it is worth noting that SPH can simulate Rayleigh Taylor instabilities and large buoyant bubbles in fluids where the density ratios are 1000:1, Grenier et al. (2009) using a momentum conserving algorithm where the pressure term is

$$
\frac{\nabla P}{\rho} \rightarrow \sum_{b} m_{b}\left(\frac{P_{a}+P_{b}}{\rho_{a} \rho_{b}}+R_{a b}+\Pi_{a b}\right) \nabla_{a} W_{a b},
$$

where $R_{a b}$ is a term which increases the pressure between the particles of different fluids by a fraction 0.08 . The algorithm due to Grenier et al. (2009)) is rather complicated and simpler algorithms are now available.

\subsection{Initial conditions}

Let us assume then that we can solve the set of equations needed for star formation. The next question is whether or not we have the right initial conditions. One view is that the final results are independent of the initial conditions. The other is that the 


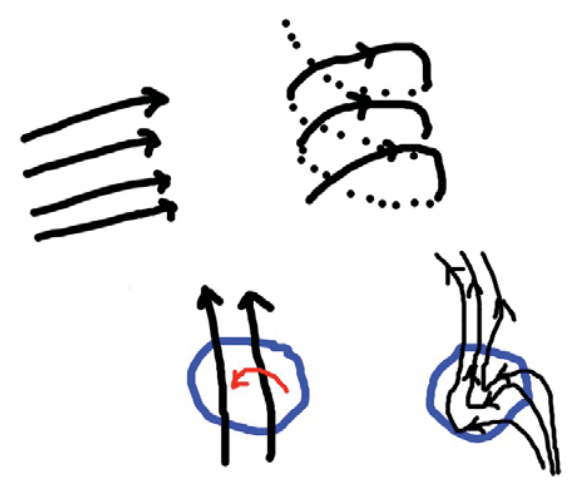

Figure 1. On the upper left is shown a sketch of a typical estimate of the magnetic field from observations, but an alternative magnetic field is that shown on the right. The fields may be twisted by differential rotation as shown in the lower sketches and such a field may be more relevant for the initial conditions for star formation.

results depend significantly on these conditions. Both views have representatives at this conference. My view is that at least some aspects of the initial conditions are important and our knowledge of galaxies should enable us to pin them down. For example, it is clear that molecular clouds forming in the galaxy will have significant rotation, which may or may not be controlled by magnetic fields. Suppose the rotation dominates the local magnetic field. The magnetic field will then be rapidly twisted by the shear (see the lower part of figure 1). At present this combination of rotation and magnetic field is disregarded as an initial condition. Instead the initial conditions are usually chosen to be a turbulent velocity field (see below) with an initial magnetic field which consists of straight field lines.

The observer's also tend to interpret fields in terms of a simple topology as shown in figure 1, though that topology could just as easily be interpreted in terms of a helical field with only one side of the helix observed (as shown in the upper right hand side of figure 1. Where we can observe magnetic fields in turbulent regions such as sunspots we find they are complicated tangles of fields even though their pressure far exceeds the thermal pressure of the gas. The final resolution will come from simulations of the formation of giant molecular clouds along the lines of the work of Claire Dobbs and Ian Bonnell (this conference) but with magnetic fields.

\subsection{Boundary conditions}

Star forming regions such as Orion appear to be independent of neighbouring star forming regions. It is therefore appropriate to consider them as isolated clouds with boundary conditions that are nearly stress free. There is a dangerous tendency however, to replace the universe by periodic boxes without testing how big the box should be before the simulation is affected by the periodicity. Detailed calculations of turbulence in nearly incompressible fluids for example, show that only a small fraction, typically 0.01 of the volume sustains isotropic turbulence. Correlation functions are also compromised by the fact that for a periodic box the corners are perfectly correlated. The conclusion is that the periodic box has to be very much larger than the scale of the processes of interest if it is to mimic an infinite fluid with satisfactory accuracy.

It is known from turbulence calculations that the turbulence is strongly affected by whether or not the boundaries are periodic, no slip, or stress free. The left frame of figure 2 shows the vorticity in a two dimensional turbulent liquid in a periodic box as calculated by Clercx et al. (2001). The vorticity has one sign in the upper left corner and the lower 

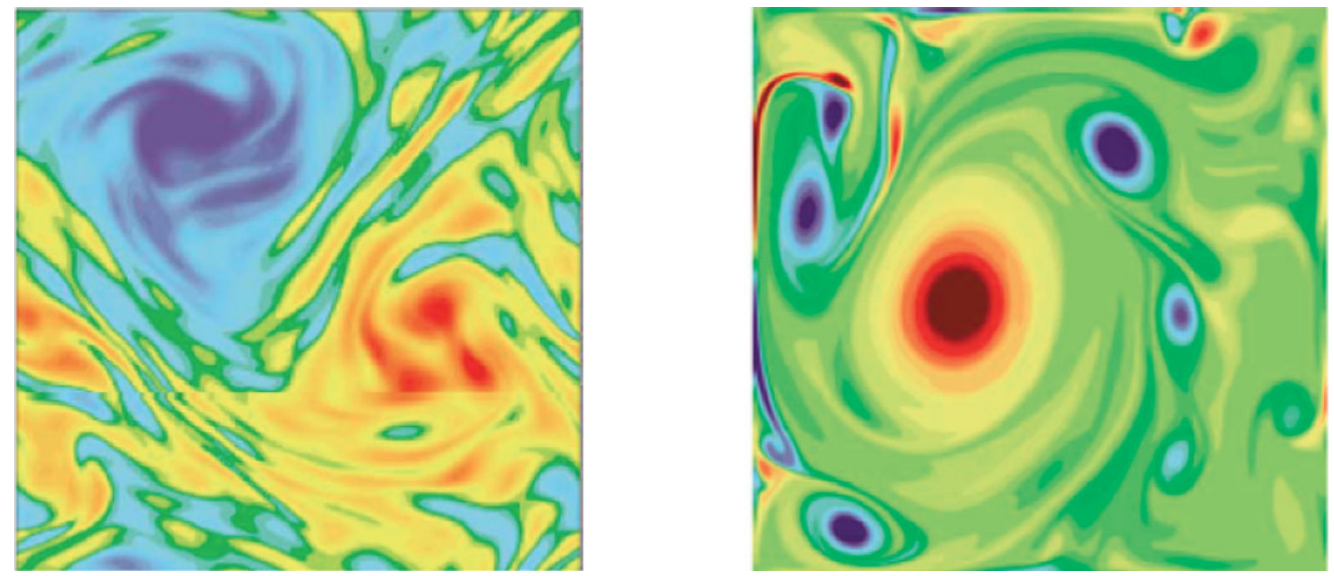

Figure 2. Decaying turbulence in a square box. The left frame shows the vorticity when the boundaries are periodic. There is vorticity of one sign in the upper left hand corner while vorticity of opposite sign occupies the lower right corner. The right frame is for the same initial conditions but with no-slip boundaries. Vorticity of one sign forms a large island surrounded by small islands of opposite vorticity. Note that in black and white the vorticity in the central part of the large circular vortex appears to be the same as that of the small islands, but it is, in fact, the peak value of the vorticity in the large island.

right corner has the opposite sign. In the right frame of figure 2 the simulation uses the same initial conditions but no-slip boundaries. The distribution of vorticity is now completely different. It consists of a large island with vorticity ringed by vorticity of the opposite sign. Similar differences show up with stress-free boundaries and boundaries of different shape e.g. rectangles.

Returning to the initial conditions we can ask what turbulence do we assign to the fluid. The answer will depend on the density distribution and boundary conditions. If they are stress free and the density decreases strongly near the boundary the turbulence will be different from that with periodic boundaries and uniform initial density. In my view periodic boundaries should not be used, or if they are used, they should be tested by comparing results for a range of box sizes.

Another aspect of turbulence which is useful to consider is that while the simulators take the view that the turbulence is largely due to shocks, the situation is much more complicated in reality. The generation of turbulence can come from Kelvin-Helmholtz instabilities, or from explosive outbursts from supernovae or indeed from any unstable fluid process. Furthermore, if there is turbulence at all scales, the shocks themselves will be affected because of turbulent mixing of momentum and thermal energy. I expect to see more work on how turbulence affects the simulation of shocks. One obvious aspect is that the shock profiles will be smoothed and the shock dissipation altered.

\subsection{Mixing}

The extent of mixing becomes important when we need to know the distribution of heavy elements or dust in order to calculate radiative transfer correctly. The extent of mixing depends on the velocity distribution and the scale over which we wish to specify the mixing. The smaller the scale the less likely it is to be well mixed. Furthermore it is known that there can be coherent structures called lagrangian coherent structures within which there is very little mixing. As a consequence rules of thumb like' the mixing will be nearly complete after two turnover times ', are false. No one at this conference has 
explored mixing carefully, and it would be useful to do this once we can simulate the ejection of heavy elements into the star forming region from supernova.

\subsection{Radiative transfer}

It was good to see the great strides that have been taken in calculating radiative transfer within gas dynamic calculations whether or not particles or grids are used. In some cases (Bate, Krumholz, Walch, Susa, Offner, Harper-Clark, Ercolano, Commercon in this conference) flux limited diffusion approximations have been used. In others (Norlund this conference) direct solution of radiative transfer by ray tracing algorithms has been achieved. Their results show how important it is to include radiative transfer because it affects the pressure terms and the extent of heating, cooling and the atomic state of the gas. The remarks made earlier about mixing of heavy elements and grains are of fundamental importance for the accuracy of radiative transfer because they change the opacity. If I can have one wish granted it would be to see a ray tracing code for SPH which uses the particles directly without recourse to a grid.

\section{A set of final questions}

To end here are a set of questions which might be asked if we disappeared and returned in 10 years. Everyone will have their own set of questions.

- Have we got reliable accurate codes that simulate gas magnetic fields, dust, ions, electrons, radiative transfer and chemistry?

- Do they predict almost everything the observers see?

- Do we have agreement on the initial and boundary conditions?

- Do we have a simple, phenomenological rule for star formation in cosmological simulations?

- If Bruce Elmegreen is right about hardware can we simulate seamlessly from GMC to stars?

- Have the observer's stopped observing because there is nothing new to see?

Given the progress shown by the work described at this conference I expect the answers to be at least a qualified yes to each question except, perhaps, the last.

\section{References}

Clercx, H. H., Nielson, A. H., Torres, D. J., \& Coutsias, E. A. 2001, Euro. J. Mech-B/Fluids,20, 557

Fowler, W. A. \& Hoyle, F. 1962, Roy. Obs. Bulletins. Her Majesty's Stationary Office., Number 67

Grenier, N., Antuono, M., Colagrossi, A., LeTouzé, , D., \& Alessandrini, B. 2009, J. Computat. Phys, 228, 8380

Monaghan, J. J. 2005, Rep. Prog. Phys., 68, 1703 